International Journal of Pure and Applied Mathematics

Volume 107 No. 1 2016, 267-278

ISSN: 1311-8080 (printed version); ISSN: 1314-3395 (on-line version)

url: http://www.ijpam.eu

doi: 10.12732/ijpam.v107i1.19

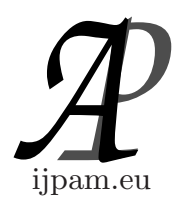

\title{
$L$-FUZZY QUASI-UNIFORM SPACES INDUCED BY L-FUZZY INTERIOR SPACES
}

Young Sun $\mathrm{Kim}^{1}$, Yong Chan $\mathrm{Kim}^{2} \S$

${ }^{1}$ Department of Applied Mathematics

Pai Chai University

Dae Jeon, 302-735, KOREA

${ }^{2}$ Department of Mathematics

Gangneung-Wonju University

Gangneung, Gangwondo, 210-702, KOREA

\begin{abstract}
In this paper, we introduce the notions of $L$-fuzzy quasi-uniformities and $L$ fuzzy interior operators in complete residuated lattices. We investigate the $L$-fuzzy quasiuniformities induced by $L$-fuzzy interior operators. We study the relationships between $L$ fuzzy interior operators and $L$-fuzzy quasi-uniformities. We give their examples.
\end{abstract}

AMS Subject Classification: 03E72, 06A15, 06F07, 54F05

Key Words: complete residuated lattices, $L$-fuzzy interior operators, $L$-fuzzy quasi-uniformities, $L F$ - interior map, $L F$-uniformly continuous

\section{Introduction}

Many researcher introduced the notion of fuzzy uniformities in unit interval $[0,1][1]$, complete distributive lattices [3,8,9], commutative unital quantales [6] and complete quasi-monoidal lattices [15].

Kim [9] introduced the notion of fuzzy quasi-uniformities as an extension of Lowen in strictly two-sided, commutative quantales. Ramadan $[12,13]$ investigated the relations among the families of $L$-fuzzy topology, $L$-neighborhood system, $L$-fuzzy topogenous structures and $L$-fuzzy quasi-uniformity.

Received: November 11, 2015

Published: March 31, 2016

$\S$ Correspondence author (c) 2016 Academic Publications, Ltd.

url: www.acadpubl.eu 
In this paper, we introduce the notions of $L$-fuzzy interior operators and $L$ fuzzy quasi-uniformities in complete residuated lattices [5]. We investigate the $L$-fuzzy quasi-uniformities induced by $L$-fuzzy interior operators. We study the relationships between $L$-fuzzy interior operators and $L$-fuzzy quasi-uniformities. We give their examples.

\section{Preliminaries}

Definition 1. $[2,5,6,15]$ An algebra $(L, \wedge, \vee, \odot, \rightarrow, 0,1)$ is called a complete residuated lattice if it satisfies the following conditions:

(C1) $L=(L, \leq, \vee, \wedge, 1,0)$ is a completely distributive with the greatest element 1 and the least element 0 ;

(C2) $(L, \odot, 1)$ is a commutative monoid;

(C3) $x \odot y \leq z$ iff $x \leq y \rightarrow z$ for $x, y, z \in L$.

In this paper, we assume that $\left(L, \leq, \odot, \rightarrow, \oplus,{ }^{*}\right)$ is a complete residuated lattice with an order reversing involution $x^{*}=x \rightarrow 0$ which is defined by $x \oplus y=\left(x^{*} \odot y^{*}\right)^{*}$ unless otherwise specified and we denote $L_{0}=L-\{0\}$.

Lemma 2. $[2,5,6,15]$ For each $x, y, z, x_{i}, y_{i}, w \in L$, we have the following properties.

(1) $1 \rightarrow x=x, 0 \odot x=0$,

(2) If $y \leq z$, then $x \odot y \leq x \odot z, x \oplus y \leq x \oplus z, x \rightarrow y \leq x \rightarrow z$ and $z \rightarrow x \leq y \rightarrow x$,

(3) $x \odot\left(\bigvee_{i} y_{i}\right)=\bigvee_{i}\left(x \odot y_{i}\right)$,

(4) $x \rightarrow\left(\bigwedge_{i} y_{i}\right)=\bigwedge_{i}\left(x \rightarrow y_{i}\right)$ and $\left(\bigvee_{i} x_{i}\right) \rightarrow y=\bigwedge_{i}\left(x_{i} \rightarrow y\right)$,

(5) $x \rightarrow\left(\bigvee_{i} y_{i}\right) \geq \bigvee_{i}\left(x \rightarrow y_{i}\right)$ and $\left(\bigwedge_{i} x_{i}\right) \rightarrow y \geq \bigvee_{i}\left(x_{i} \rightarrow y\right)$,

(6) $(x \odot y) \rightarrow z=x \rightarrow(y \rightarrow z)=y \rightarrow(x \rightarrow z)$,

(7) $x \odot(x \rightarrow y) \leq y$ and $x \rightarrow y \leq(y \rightarrow z) \rightarrow(x \rightarrow z)$,

(8) $(x \rightarrow y) \odot(z \rightarrow w) \leq(x \odot z) \rightarrow(y \odot w)$,

(9) $(x \rightarrow y) \odot(z \rightarrow w) \leq(x \oplus z) \rightarrow(y \oplus w)$,

(10) $x \rightarrow y \leq(x \odot z) \rightarrow(y \odot z)$ and $(x \rightarrow y) \odot(y \rightarrow z) \leq x \rightarrow z$,

(11) $x \rightarrow y=y^{*} \rightarrow x^{*}$.

Definition 3. [6,13] A mapping int : $L^{X} \times L_{0} \rightarrow L^{X}$ is called an $L$ - fuzzy interior operator if it satisfies the following conditions;

(I1) $\operatorname{int}\left(1_{X}, r\right)=1_{X}$,

(I2) $\operatorname{int}(\lambda, r) \leq \lambda$,

(I3) If $\lambda_{1} \leq \lambda_{2}$, then $\left.\operatorname{int}\left(\lambda_{1}, r\right) \leq \operatorname{int}\left(\lambda_{2}, r\right)\right)$, 
(I4) If $r_{1} \leq r_{2}$, then $\operatorname{int}\left(\lambda, r_{1}\right) \geq \operatorname{int}\left(\lambda, r_{2}\right)$,

(I5) $\operatorname{int}\left(\lambda_{1} \odot \lambda_{2}, r \odot s\right) \geq \operatorname{int}\left(\lambda_{1}, r\right) \odot \operatorname{int}\left(\lambda_{2}, s\right)$.

The pair $(X, i n t)$ is called an $L$-fuzzy interior space.

Let $\left(X, i n t_{X}\right)$ and $\left(Y, i n t_{Y}\right)$ be $L$-fuzzy interior spaces and $f: X \rightarrow Y$ be a map. Then $f$ is called an $L F$ - interior map if, for each $\rho \in L^{Y}, r \in L_{0}$,

$$
\operatorname{int}_{X}\left(f^{\leftarrow}(\rho), r\right) \geq f^{\leftarrow}\left(\operatorname{int}_{Y}(\rho, r)\right) .
$$

Definition 4. $\quad[9,13]$ A mapping $\mathcal{U}: L^{X \times X} \rightarrow L$ is called an $L$-fuzzy quasi-uniformity on $X$ iff it satisfies the conditions.

(QU1) There exists $u \in L^{X \times X}$ such that $\mathcal{U}(u)=1$,

(QU2) If $v \leq u$, then $\mathcal{U}(v) \leq \mathcal{U}(u)$,

(QU3) For every $u, v \in L^{X \times X}, \quad \mathcal{U}(u \odot v) \geq \mathcal{U}(u) \odot \mathcal{U}(v)$,

(QU4) If $\mathcal{U}(u) \neq 0$, then $1_{\triangle} \leq u$, where

$$
1_{\Delta}(x, y)= \begin{cases}1 & \text { if } x=y \\ 0 & \text { if } x \neq y\end{cases}
$$

(QU5) $\bigvee\{\mathcal{U}(v) \mid v \circ v \leq u\} \geq \mathcal{U}(U)$,

$$
v \circ w(x, z)=\bigvee_{z \in X}(v(y, z) \odot w(x, y)), \quad \forall x, y \in X
$$

The pair $(X, \mathcal{U})$ is called an $L$-fuzzy quasi-uniform space.

Let $\mathcal{U}_{X}$ and $\mathcal{U}_{Y}$ be $L$-fuzzy quasi-uniformities on $X$ and $Y$, respectively and $f: X \rightarrow Y$ be a map. Then $f$ is called $L F$-uniformly continuous if

$$
\mathcal{U}_{Y}(v) \leq \mathcal{U}_{X}\left((f \times f)^{\leftarrow}(v)\right), \forall v \in L^{Y \times Y}
$$

Remark 5. Let $\mathcal{U}$ be an $L$-fuzzy quasi-uniformity on $X$. Then by (QU1) and (QU2), we have $\mathcal{U}\left(1_{X \times X}\right)=1$ because $u \leq 1_{X \times X}$ for all $u \in L^{X \times X}$.

\section{L-Fuzzy Quasi-Uniform Spaces Induced by $L$-Fuzzy Interior Spaces}

Theorem 6. Let $(X, \mathcal{U})$ be an $L$-quasi-uniform space. Define mappings int $_{\mathcal{U}}: L^{X} \times L_{0} \rightarrow L^{X}$ as

$$
\operatorname{int}_{\mathcal{U}}(\lambda, r)=\bigvee\{\vec{u}\{\lambda\} \mid \mathcal{U}(u) \geq r\}
$$


where

$$
\vec{u}\{\lambda\}(y)=\bigwedge_{x \in X}(u(y, x) \rightarrow \lambda(x)) .
$$

We have the following properties.

(1)

$$
\begin{aligned}
& \bigwedge_{x \in X}\left(\operatorname{int}_{\mathcal{U}}\left(\lambda_{1}, r\right)(x) \rightarrow \operatorname{int}_{\mathcal{U}}\left(\lambda_{2}, r\right)(x)\right) \\
& \geq \bigwedge_{y \in X}\left\{\lambda_{1}(y) \rightarrow \lambda_{2}(y)\right\} .
\end{aligned}
$$

(2) int $_{\mathcal{U}}$ is an $L$-fuzzy interior operator.

(3) $\operatorname{int}_{\mathcal{U}}\left(\operatorname{int}_{\mathcal{U}}\left(\lambda, r_{1}\right), r_{1}\right) \geq \operatorname{int}_{\mathcal{U}}(\lambda, r)$ for each $r_{1}<r$.

Proof. (1)

$$
\begin{aligned}
& \bigwedge_{x \in X}\left(\operatorname{int}_{\mathcal{U}}\left(\lambda_{1}, r\right)(x) \rightarrow \operatorname{int}_{\mathcal{U}}\left(\lambda_{2}, r\right)(x)\right) \\
& =\bigwedge_{x \in X} \bigvee\left\{\left(\bigwedge_{y \in X} u(x, y) \rightarrow \lambda_{1}(y)\right) \mid \mathcal{U}(u) \geq r\right\} \\
& \left.\rightarrow \bigwedge_{y \in X}\left(u(x, y) \rightarrow \lambda_{2}(y)\right) \mid \mathcal{U}(u) \geq r\right\} \\
& \geq \bigwedge_{x \in X} \bigvee \bigwedge_{y \in X}\left\{\left(u(x, y) \rightarrow \lambda_{1}(y)\right) \rightarrow\left(u(x, y) \rightarrow \lambda_{2}(y)\right) \mid \mathcal{U}(u) \geq r\right\} \\
& \geq \bigwedge_{y \in X}\left\{\lambda_{1}(y) \rightarrow \lambda_{2}(y)\right\} .
\end{aligned}
$$

(2) (I1) Since $u(x, y) \rightarrow 1_{X}(x)=1$, int $\left.\mathcal{U}_{\mathcal{U}}\left(1_{X}, A\right), r\right)=\left(1_{X}, A\right)$.

(I2) Since

$$
\vec{u}\{\lambda\}(y)=\bigwedge_{x \in X}(u(y, x) \rightarrow \lambda(x)) \leq u(y, y) \rightarrow \lambda(y)=\lambda(y),
$$

then $\vec{u}\{\lambda\} \leq \lambda$. Hence $\operatorname{int}_{\mathcal{U}}(\lambda, r) \leq \lambda$.

(I3) By (1), if $\lambda_{1} \leq \lambda_{2}$, then $\operatorname{int}_{\mathcal{U}}\left(\lambda_{1}, r\right) \leq \operatorname{int}_{\mathcal{U}}\left(\lambda_{2}, r\right)$.

(I4) It is obvious from the definition of int . $_{\text {. }}$

(I5) Since $\left(u(x, y) \rightarrow \lambda_{1}(x)\right) \odot\left(v(x, y) \rightarrow \lambda_{2}(x)\right) \leq u(x, y) \odot v(x, y) \rightarrow$ $\left(\lambda_{1} \odot \lambda_{2}(x)\right.$,

$$
\begin{aligned}
& \operatorname{int}_{\mathcal{U}}\left(\lambda_{1}, r\right) \odot \operatorname{int}_{\mathcal{U}}\left(\lambda_{2}, s\right) \\
& =\bigvee\left\{\bigwedge_{x \in X}\left(u(y, x) \rightarrow \lambda_{1}(x)\right) \mid \mathcal{U}(u) \geq r\right\} \\
& \odot \bigvee\left\{\bigwedge_{x \in X}\left(v(y, x) \rightarrow \lambda_{2}(x)\right) \mid \mathcal{U}(v) \geq s\right\} \\
& \leq \bigvee\left\{\bigwedge_{x \in X}\left((u(y, x) \odot v(y, x)) \rightarrow\left(\lambda_{1} \odot \lambda_{2}\right)(x)\right) \mid \mathcal{U}(u \odot v) \geq r \odot s\right\} \\
& \leq \operatorname{int}_{\mathcal{U}}\left(\lambda_{1} \odot \lambda_{2}, r \odot s\right),
\end{aligned}
$$

(3) Suppose that there exists $\lambda \in L^{X}$ and $r, s \in L_{0}$ with $r>s$ such that

$$
\operatorname{int}_{\mathcal{U}}\left(\operatorname{int}_{\mathcal{U}}(\lambda, s), s\right) \nsupseteq \operatorname{int}_{\mathcal{U}}(\lambda, r) .
$$

From the definition of $\operatorname{int}_{\mathcal{U}}(\lambda, r)$, there exists $u \in S(X \times X, A)$ with $\mathcal{U}(u) \geq r$ such that

$$
\operatorname{int}_{\mathcal{U}}\left(\operatorname{int}_{\mathcal{U}}(\lambda, s), s\right) \nsupseteq \bigwedge_{x \in X} u(-, x) \rightarrow \lambda(x) .
$$


On the other hand, since $\bigvee\{\mathcal{U}(v) \mid v \circ v \leq u\} \geq \mathcal{U}(u) \geq r$, for each $s<r$, there exists $v \in L^{X \times X}$ with $v \circ v \leq u$ and $\mathcal{U}(v) \geq s$.

$$
\begin{aligned}
& \operatorname{int}_{\mathcal{U}}\left(\operatorname{int}_{\mathcal{U}}(\lambda, s), s\right)(y) \\
& =\bigvee\left\{\bigwedge_{x \in X}\left(w(y, x) \rightarrow \operatorname{int}_{\mathcal{U}}(\lambda, s)(x)\right) \mid \mathcal{U}(w) \geq s\right\} \\
& \geq \bigwedge_{x \in X}\left(v(y, x) \rightarrow \bigwedge_{z \in X}(v(x, z) \rightarrow \lambda(z))\right) \\
& =\bigwedge_{x, z \in X}((v(y, x) \odot v(x, z)) \rightarrow \lambda(z)) \\
& =\bigwedge_{z \in X}\left(\bigvee_{x \in X}(v(y, x) \odot v(x, z)) \rightarrow \lambda(z)\right) \\
& \geq \bigwedge_{z \in X}(u(y, z) \rightarrow \lambda(z))
\end{aligned}
$$

It is a contradiction. Hence the result hold.

Lemma 7. For every $\lambda \in L^{X}$, we define $u_{\lambda}, u_{\lambda}^{-1}: L^{X \times X} \rightarrow L$ by

$$
\begin{gathered}
u_{\lambda}(x, y)=\lambda(x) \rightarrow \lambda(y) \\
u_{\lambda}^{-1}(x, y)=u_{\lambda}(y, x)
\end{gathered}
$$

then we have the following statements

(1) $1_{X \times X}=u_{0_{X}}=u_{1_{X}}$ and $1_{\triangle} \leq u_{\lambda}$,

(2) For every $u_{\rho} \in L^{X \times X}$, we have $u_{\rho} \circ u_{\rho} \leq u_{\rho}$,

(3) $u_{\lambda} \odot u_{\rho} \leq u_{\lambda \odot \rho}$ and $u_{\lambda} \odot u_{\rho} \leq u_{\lambda \oplus \rho}$,

(4) $u_{\rho}^{-1}=u_{\rho^{*}}$ and $u_{\lambda \odot \rho}^{-1}=u_{\lambda^{*} \oplus \rho^{*}}$.

Proof. (1)

$$
\begin{aligned}
& 1_{X \times X}(x, y)=1=u_{0_{X}}(x, y)=0_{X}(x) \rightarrow 0_{X}(y) \\
& =1_{X}(x) \rightarrow 1_{X}(y)=u_{1_{X}}(x, y) .
\end{aligned}
$$

Since $u_{\lambda}(x, x)=\lambda(x) \rightarrow \lambda(x)=1,1_{\triangle} \leq u_{\lambda}$.

(2)

$$
\begin{aligned}
& \left(u_{\rho} \circ u_{\rho}\right)(x, z)=\bigvee_{y \in X}\left(u_{\rho}(x, y) \odot u_{\rho}(y, z)\right) \\
& =\bigvee_{y \in X}((\rho(x) \rightarrow \rho(y)) \odot(\rho(y) \rightarrow \rho(z)) \\
& =\rho(x) \rightarrow \rho(z)=u_{\rho}(x, z)
\end{aligned}
$$

(3)

$$
\begin{aligned}
& \left(u_{\lambda} \odot u_{\rho}\right)(x, y)=u_{\lambda}(x, y) \odot u_{\rho}(x, y)=(\lambda(x) \rightarrow \lambda(y)) \odot(\rho(x) \rightarrow \rho(y)) \\
& \leq \lambda(x) \odot \rho(x) \rightarrow \lambda(y) \odot \rho(y)=u_{\lambda \odot \rho}(x, y) . \\
& \left(u_{\lambda} \odot u_{\rho}\right)(x, y)=u_{\lambda}(x, y) \odot u_{\rho}(x, y)=(\lambda(x) \rightarrow \lambda(y)) \odot(\rho(x) \rightarrow \rho(y) \\
& \leq \lambda(x) \oplus \rho(x) \rightarrow \lambda(y) \oplus \rho(y)=u_{\lambda \oplus \rho}(x, y) .
\end{aligned}
$$


(4)

$$
\begin{aligned}
& u_{\lambda}^{-1}(x, y)=u_{\lambda}(y, x)=\lambda(y) \rightarrow \lambda(x)=\lambda^{*}(x) \rightarrow \lambda^{*}(y)=u_{\lambda^{*}}(x, y) . \\
& u_{\lambda \odot \rho}^{-1}=u_{(\lambda \odot \rho)^{*}}=u_{\lambda^{*} \oplus \rho^{*}}
\end{aligned}
$$

In the following theorem, we obtain an $L$-fuzzy quasi-uniform structure from an $L$-fuzzy interior operator.

Theorem 8. Let $(X, i n t)$ be an $L$-fuzzy interior space. Define $\mathcal{U}_{\text {int }}$ : $L^{X \times X} \rightarrow L$ by

$$
\begin{aligned}
& \mathcal{U}_{\text {int }}(u) \\
& =\bigvee\left\{\odot_{i=1}^{n} r_{i} \mid \lambda_{i}=\operatorname{int}\left(\lambda_{i}, r_{i}\right), \odot_{i=1}^{n} u_{\lambda_{i}} \leq u\right\}
\end{aligned}
$$

where $\bigvee$ is taken over every finite family $\left\{u_{\lambda_{i}} \mid i=1,2,3, \ldots, n\right\}$. Then we have the following properties.

(1) $\mathcal{U}_{\text {int }}$ is an $L$-fuzzy quasi-uniformity on $X$.

(2) If $\operatorname{int}(\operatorname{int}(\lambda, r), r)=\operatorname{int}(\lambda, r)$, then $i n t_{\mathcal{U}_{i n t}} \geq i n t$.

(3) If $\mathcal{U}$ is an $L$-fuzzy quasi-uniformity on $X$ such that $\mathcal{U}\left(\bigwedge_{i \in \Gamma} u_{i}\right)=$ $\bigwedge_{i \in \Gamma} \mathcal{U}\left(u_{i}\right)$ for each $u_{i}$ and $i \in \Gamma$, then $\mathcal{U}_{i n t_{\mathcal{U}}}(u) \leq \mathcal{U}(u)$.

Proof. (1) (QU1) Since $\operatorname{int}\left(1_{X}, r\right)=1_{X}, \operatorname{cl}\left(0_{X}, r\right)=0_{X}$, there exists $1_{X \times X}=u_{0_{X}}=u_{1_{X}} \in L^{X \times X}$. It follows $\mathcal{U}_{\text {int }}\left(1_{X \times X}\right)=1$.

(QU2) It is trivial from the definition of $\mathcal{U}_{\text {int }}$.

(QU3) Suppose there exist $u, v \in L^{X \times X}$ such that

$$
\mathcal{U}_{\text {int }}(u \odot v) \nsupseteq \mathcal{U}_{\text {int }}(u) \odot \mathcal{U}_{\text {int }}(v) .
$$

There exist two finite families $\left\{\lambda_{i} \in L^{X} \mid \operatorname{int}\left(\lambda_{i}, r_{i}\right)=\lambda_{i}, \odot_{i=1}^{m} u_{\lambda_{i}} \leq u\right\}$ and $\left\{\rho_{j} \in L^{X} \mid \operatorname{int}\left(\rho_{j}, s_{j}\right)=\rho_{j}, \odot_{j=1}^{n} u_{\rho_{j}} \leq v\right\}$ such that

$$
\mathcal{U}_{\text {int }}(u \odot v) \nsupseteq\left(\odot_{i=1}^{m} r_{i}\right) \odot\left(\odot_{j=1}^{n} s_{j}\right) .
$$

On the other hand, since $u \odot v \geq\left(\odot_{i=1}^{m} u_{\lambda_{i}}\right) \odot\left(\odot_{j=1}^{n} u_{\rho_{j}}\right)$ and,

$$
\left(\odot_{i=1}^{m} \operatorname{int}\left(\lambda_{i}, r_{i}\right) \odot\left(\odot_{j=1}^{n} i n t\left(\rho_{j}, s_{j}\right)\right)=\left(\odot_{i=1}^{m} \lambda_{i}\right) \odot\left(\odot_{j=1}^{n} \rho_{j}\right)\right.
$$

we have

$$
\mathcal{U}_{i n t}(u \odot v) \geq\left(\odot_{i=1}^{m} r_{i}\right) \odot\left(\odot_{j=1}^{n} s_{j}\right)
$$

It is a contradiction. 
(QU4) Let $\mathcal{U}_{\text {int }}(u) \neq 0$. Then there exists a finite family $\left\{\lambda_{i} \in L^{X} \mid\right.$ $\left.\operatorname{int}\left(\lambda_{i}, r_{i}\right)=\lambda_{i}, \odot_{i=1}^{m} u_{\lambda_{i}} \leq u\right\}$ such that

$$
\mathcal{U}_{\text {int }}(u)=\odot_{i=1}^{n} r_{i} \neq 0 .
$$

Since $u_{\lambda_{i}} \geq 1_{\triangle}$ from Lemma $7(1)$,

$$
1_{\triangle} \leq \odot_{i=1}^{m} u_{\lambda_{i}} \leq u
$$

(QU5) Suppose there exists $u \in L^{X \times X}$ such that

$$
\bigvee\left\{\mathcal{U}_{\text {int }}(v) \mid v \circ v \leq u\right\} \nsupseteq \mathcal{U}_{\text {int }}(u) .
$$

There exists a finite family $\left\{\rho_{i} \in L^{X \times X} \mid \operatorname{int}\left(\rho_{i}, r_{i}\right)=\rho_{i}, \odot_{i=1}^{m} u_{\rho_{i}} \leq u\right\}$ such that

$$
\bigvee\left\{\mathcal{U}_{i n t}(v) \mid v \circ v \leq u\right\} \gtreqless \odot_{i=1}^{m} r_{i} .
$$

On the other hand, since $u_{\rho_{i}} \circ u_{\rho_{i}}=u_{\rho_{i}}$ for each $i \in\{1, \ldots, m\}$ from Lemma $7(2)$, we have $\left(\odot_{i=1}^{m} u_{\rho_{i}} \circ\left(\odot_{i=1}^{m} u_{\rho_{i}}\right) \leq \odot_{i=1}^{m} u_{\rho_{i}}\right.$ from

$$
\begin{aligned}
& \bigvee_{y \in X}\left(\left(\odot_{i=1}^{m} u_{\rho_{i}}(x, y)\right) \odot\left(\odot_{i=1}^{m} u_{\rho_{i}}(y, z)\right)\right) \\
& =\bigvee_{y \in X}\left(\left(\odot_{i=1}^{m}\left(\rho_{i}(x) \rightarrow \rho_{i}(y)\right) \odot\left(\odot_{i=1}^{m}\left(\rho_{i}(y) \rightarrow \rho_{i}(z)\right)\right)\right)\right. \\
& =\bigvee_{y \in X}\left(\left(\odot_{i=1}^{m}\left(\rho_{i}(x) \rightarrow \rho_{i}(y)\right) \odot\left(\rho_{i}(y) \rightarrow \rho_{i}(z)\right)\right)\right) \\
& \leq \odot_{i=1}^{m}\left(\rho_{i}(x) \rightarrow \rho_{i}(z)\right) .
\end{aligned}
$$

Put $v=\odot_{i=1}^{m} u_{\rho_{i}}$. Since $\operatorname{int}\left(\rho_{i}, r_{i}\right)=\rho_{i}, \odot_{i=1}^{m} u_{\rho_{i}} \leq v, v \circ v \leq u$ and

$$
\bigvee\left\{\mathcal{U}_{i n t}(w) \mid w \circ w \leq u\right\} \geq \mathcal{U}_{i n t}(v) \geq \odot_{i=1}^{m} r_{i}
$$

It is a contradiction. Thus $\bigvee\left\{\mathcal{U}_{\text {int }}(v) \mid v \circ v \leq u\right\} \geq \mathcal{U}_{\text {int }}(u)$.

Hence $\mathcal{U}_{\text {int }}$ is an $L$-fuzzy quasi-uniformity on $X$.

(2) Let $\operatorname{int}(\operatorname{int}(\lambda, r), r)=\operatorname{int}(\lambda, r)$. Then $\mathcal{U}_{\text {int }}\left(u_{\text {int }(\lambda, r)}\right) \geq r$.

$$
\begin{aligned}
& \operatorname{int}_{\mathcal{U}_{i n t}}(\lambda, r)(y)=\bigvee\left\{\bigwedge_{x \in X}(U(y, x) \rightarrow \lambda(x)) \mid \mathcal{U}_{i n t}(v) \geq r\right\} \\
& \geq \bigwedge_{x \in X}\left(u_{i n t}(\lambda, r)(y, x) \rightarrow \lambda(x)\right) \\
& \geq \bigwedge_{x \in X}((\operatorname{int}(\lambda, r)(y) \rightarrow \operatorname{int}(\lambda, r)(x)) \rightarrow \lambda(x)) \\
& \geq \operatorname{int}(\lambda, r)(y)
\end{aligned}
$$

because

$$
\begin{aligned}
& \operatorname{int}(\lambda, r)(y) \odot(\operatorname{int}(\lambda, r)(y) \rightarrow \operatorname{int}(\lambda, r)(x)) \\
& \leq \operatorname{int}(\lambda, r)(x) \leq \lambda(x) .
\end{aligned}
$$

Hence int $_{\mathcal{U}_{\text {int }}} \geq$ int. 
(3) Suppose there exists $u \in L^{X \times X}$ such that $\mathcal{U}_{\text {int }_{\mathcal{U}}}(u) \not \mathcal{U}(u)$. By the definition of $\mathcal{U}_{\text {int }_{\mathcal{U}}}$, there exists $r_{i} \in L_{0}$ with $\operatorname{int}_{\mathcal{U}}\left(\lambda_{i}, r_{i}\right)=\lambda_{i}$ and $\odot_{i=1}^{n} u_{\lambda_{i}} \leq u$ such that $\odot_{i=1}^{n} r_{i} \not \mathbb{Z} \mathcal{U}(u)$.

On the other hand, $\operatorname{int}_{\mathcal{U}}\left(\lambda_{i}, r_{i}\right)=\bigvee_{k}\left\{\overrightarrow{w_{i_{k}}}\left\{\lambda_{i}\right\} \mid \mathcal{U}\left(w_{i_{k}}\right) \geq r_{i}\right\}$. Since $L$ is a complete distributive lattice,

$$
\bigvee_{k} \overrightarrow{w_{i_{k}}}\left\{\lambda_{i}\right\}=\bigwedge_{k} w_{i_{k}}\left\{\lambda_{i}\right\}
$$

Put $w_{i}=\bigwedge_{k} w_{i_{k}}$. Then $\mathcal{U}\left(w_{i}\right)=\mathcal{U}\left(\bigwedge_{k} w_{i_{k}}\right)=\bigwedge_{k} \mathcal{U}\left(w_{i_{k}}\right) \geq r_{i}$ and $\operatorname{int}_{\mathcal{U}}\left(\lambda_{i}, r_{i}\right)=$ $\vec{w}_{i}\left\{\lambda_{i}\right\}=\lambda_{i}$. Since $w_{i} \leq u_{\lambda_{i}}$,

$$
\mathcal{U}(u) \geq \mathcal{U}\left(\odot_{i=1}^{n} u_{\lambda_{i}}\right) \geq \odot_{i=1}^{n} \mathcal{U}\left(u_{\lambda_{i}}\right) \geq \odot_{i=1}^{n} \mathcal{U}\left(w_{i}\right) \geq \odot_{i=1}^{n} r_{i} .
$$

It is a contradiction.

Theorem 9. Let $(X, \mathcal{U})$ and $(Y, \mathcal{V})$ be $L$-fuzzy quasi uniform spaces and $f: X \rightarrow Y$ be $L F$-uniformly continuous. Then $\operatorname{int}_{\mathcal{U}}\left(f^{\leftarrow}(\rho), r\right) \geq f^{\leftarrow}(\operatorname{int} \mathcal{V}(\rho, r))$.

Proof. Since

$$
\begin{aligned}
& \overrightarrow{(f \times f)^{\leftarrow}(v)}\left\{f^{\leftarrow}(\rho)\right\}(y)=\bigwedge_{x \in X}\left((f \times f)^{\leftarrow}(v)(x, y) \rightarrow f^{\leftarrow}(\rho)(x)\right) \\
& =\bigwedge_{x \in X}(v(f(x), f(y)) \rightarrow \rho(f(x))) \geq \bigwedge_{z \in Y}(v(z, f(y)) \rightarrow \rho(z)) \\
& =f^{\leftarrow}(\vec{v}\{\rho\})(y) \text {. }
\end{aligned}
$$

we have

$$
\begin{aligned}
& f^{\leftarrow}\left(\operatorname{int}_{\mathcal{V}}(\rho, r)\right)=f^{\leftarrow}(\bigvee\{\vec{v}\{\rho\} \mid \mathcal{V}(v) \geq r\}) \\
& =\bigvee\left\{f^{\leftarrow}(\vec{v}\{\rho\}) \mid \mathcal{V}(v) \geq r\right\} \\
& \leq \bigvee\left\{\overrightarrow{(f \times f)^{\leftarrow}(v)}\left\{f^{\leftarrow}(\rho)\right\} \mid \mathcal{U}\left((f \times f)^{\leftarrow}(v)\right) \geq r\right\} \\
& \leq \operatorname{int}_{\mathcal{U}}\left(f^{\leftarrow}(\rho), r\right) .
\end{aligned}
$$

Theorem 10. Let $\left(X, i n t_{X}\right)$ and $\left(Y, i n t_{Y}\right)$ be $L$-fuzzy interior spaces and $f: X \rightarrow Y$ be $L F$-interior map. Then the mapping $f:\left(X, \mathcal{U}_{\text {int }_{X}}\right) \rightarrow\left(Y, \mathcal{U}_{\text {int }_{Y}}\right)$ is $L F$-uniformly continuous.

Proof. We have $(f \times f)^{\leftarrow}\left(u_{\rho}\right)=u_{f \leftarrow(\rho)}$ from:

$$
\begin{aligned}
& (f \times f)^{\leftarrow}\left(u_{\rho}\right)(x, y)=u_{\rho}(f(x), f(y))=\rho(f(x)) \rightarrow \rho(f(y)) \\
& =f^{\leftarrow}(\rho)(x) \rightarrow f^{\leftarrow}(\rho)(y)=u_{f^{\leftarrow}(\rho)}(x, y) .
\end{aligned}
$$

Since $f:\left(X\right.$, int $\left._{X}\right) \rightarrow\left(Y, i n t_{Y}\right)$ is an $L F$-interior map, then 


$$
\begin{aligned}
& \mathcal{U}_{\text {int }_{Y}}(v)=\bigvee\left\{\odot_{i=1}^{n} r_{i} \mid \operatorname{int}_{Y}\left(\rho_{i}, r_{i}\right)=\rho_{i}, \odot_{i=1}^{n} u_{\rho_{i}} \leq v\right\} \\
& \leq \bigvee\left\{\odot_{i=1}^{n} r_{i} \mid \text { int }_{X}\left(f^{\leftarrow}\left(\rho_{i}\right), r_{i}\right)=f^{\leftarrow}\left(\rho_{i}\right), \odot_{i=1}^{n} u_{f} \leftarrow\left(\rho_{i}\right) \leq(f \times f)^{\leftarrow}(v)\right\} \\
& \leq \bigvee\left\{\odot_{i=1}^{n} r_{i} \mid \text { int }_{X}\left(\lambda_{i}, r_{i}\right)=\lambda_{i}, \odot_{i=1}^{n} u_{\lambda_{i}} \leq(f \times f)^{\leftarrow}(v)\right\} \\
& =\mathcal{U}_{\text {int }_{X}}\left((f \times f)^{\leftarrow}(v)\right) .
\end{aligned}
$$

Example 11. Let $X=\{x, y, z\}$ be a set and $(L=[0,1], \odot, \rightarrow)$ be a residuated lattice defined as

$$
a \odot b=(a+b-1) \vee 0, a \rightarrow b=(1-a+b) \wedge 1 .
$$

(1) Put $w \in L^{X \times X}$ such that

$$
w=\left(\begin{array}{ccc}
1 & 0.5 & 0.3 \\
0.7 & 1 & 0.5 \\
0.6 & 0.6 & 1
\end{array}\right) w \odot w=\left(\begin{array}{ccc}
1 & 0 & 0 \\
0.4 & 1 & 0 \\
0.2 & 0.2 & 1
\end{array}\right)
$$

Define $\mathcal{U}: L^{X \times X} \rightarrow L$ as follows

$$
\mathcal{U}(u)= \begin{cases}1, & \text { if } u=1_{X \times X}, \\ 0.6, & \text { if } w \leq u \neq 1_{X \times X}, \\ 0.3, & \text { if } w \odot w \leq u \geq w, \\ 0, & \text { otherwise. }\end{cases}
$$

Since $w \circ w=w$ and $(w \odot w) \circ(w \odot w)=w \odot w, \mathcal{U}$ is an $L$-fuzzy quasi-uniformity on $X$. By Theorem 6, we obtain $L$-fuzzy interior operator $i n t_{\mathcal{U}}: L^{X} \times L_{0} \rightarrow L^{X}$ as follows

$$
\text { int }_{\mathcal{U}}(\lambda, r)= \begin{cases}\bigwedge_{x \in X} \lambda(x), & \text { if } r>0.6 \\ \vec{w}\{\lambda\}, & \text { if } 0.3<r \leq 0.6 \\ \overrightarrow{w \odot w}\{\lambda\}, & \text { if } 0<r \leq 0.3,\end{cases}
$$

By Theorem 8, we obtain $\mathcal{U}_{\text {int }_{\mathcal{U}}}: L^{X \times X} \rightarrow L$ as follows

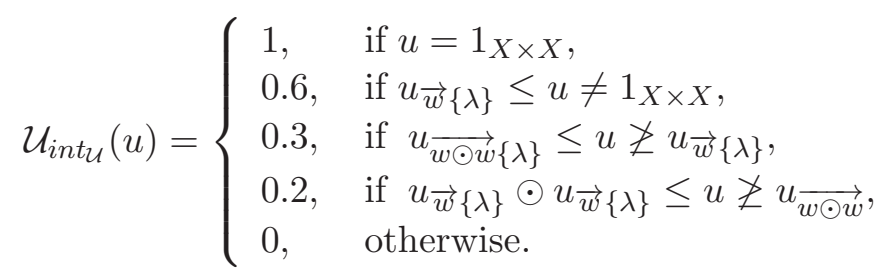


Since $w(x, y) \leq \vec{w}\{\lambda\}(x) \rightarrow \vec{w}\{\lambda\}(y)$ from

$$
\begin{aligned}
& w(y, p) \odot w(x, y) \odot \vec{w}\{\lambda\}(x) \\
& \leq w(x, p) \odot \bigwedge_{z \in X}(w(x, z) \rightarrow \lambda(z)) \\
& \leq w(x, p) \odot(w(x, p) \rightarrow \lambda(p)) \leq \lambda(p) \\
& \text { iff } w(x, y) \odot \vec{w}\{\lambda\}(x) \leq w(y, p) \rightarrow \lambda(p) \\
& \text { iff } w(x, y) \leq \vec{w}\{\lambda\}(x) \rightarrow \vec{w}\{\lambda\}(y) .
\end{aligned}
$$

Hence $\mathcal{U}_{\text {int }_{\mathcal{U}}}(u) \leq \mathcal{U}(u)$ for all $u \in L^{X \times X}$.

(2) Define a $[0,1]$-fuzzy interior operator int : $[0,1]^{X} \times(0,1] \rightarrow[0,1]^{X}$ as follows:

$$
\operatorname{int}(\lambda, r)= \begin{cases}1_{X}, & \text { if } \lambda=1_{X}, r \in[0,1], \\ \rho, & \text { if } \rho \leq \lambda, r \leq 0.6, \\ \rho \odot \rho & \text { if } \rho \odot \rho \leq \lambda \geq \rho, r \leq 0.3 \\ 0_{X}, & \text { otherwise. }\end{cases}
$$

We have $\operatorname{int}(\operatorname{int}(\lambda, r), r)=\operatorname{int}(\lambda, r)$.

By Theorem 8 , we can obtain $\mathcal{U}_{\text {int }}:[0,1]^{X \times X} \rightarrow[0,1]$ as follows:

$$
\begin{aligned}
& \mathcal{U}_{\text {int }}(u)= \begin{cases}1, & \text { if } u=u_{1_{X \times X}} \\
0.6, & \text { if } u \geq u_{\rho}, u \nsupseteq u_{\rho \odot \rho,} \\
0.3, & \text { if } u \geq u_{\rho \odot \rho}, u \geq u_{\rho}, \\
0.2, & \text { if } u \geq u_{\rho} \odot u_{\rho}, u \geq u_{\rho \odot \rho}, \\
0, & \text { otherwise. }\end{cases} \\
& u_{\rho}=\left(\begin{array}{ccc}
1 & 0.6 & 1 \\
1 & 1 & 1 \\
0.8 & 0.4 & 1
\end{array}\right) u_{\rho \odot \rho}=\left(\begin{array}{ccc}
1 & 1 & 1 \\
1 & 1 & 1 \\
0.6 & 0.6 & 1
\end{array}\right) \cdot \\
& u_{\rho} \odot u_{\rho}=\left(\begin{array}{ccc}
1 & 0.2 & 1 \\
1 & 1 & 1 \\
0.6 & 0 & 1
\end{array}\right) \cdot
\end{aligned}
$$

From Theorem 6,

$$
\operatorname{int}_{\mathcal{U}_{i n t}}(\lambda, r)(y)=\bigvee\left\{\bigwedge_{y \in X}(u(y, x) \rightarrow \lambda(x)) \mid \mathcal{U}_{i n t}(u) \geq r\right\}
$$

For $0.6<r$, int $\operatorname{U}_{\text {int }}(\lambda, r)=\bigwedge_{y \in X}\left(1_{X \times X}(y, x) \rightarrow \lambda(x)\right)=$

$$
\left(\begin{array}{rl}
\lambda(x) & \wedge \lambda(y) \wedge \lambda(z) \\
\lambda(x) & \wedge \lambda(y) \wedge \lambda(z) \\
\lambda(x) & \wedge \lambda(y) \wedge \lambda(z)
\end{array}\right)
$$


For $0.3<r \leq 0.6$, int $_{\mathcal{U}_{\text {int }}}(\lambda, r)=\bigwedge_{y \in X}\left(u_{\rho}(y, x) \rightarrow \lambda(x)\right)=$

$$
\left(\begin{array}{rl}
\lambda(x) & \wedge(0.4+\lambda(y)) \wedge \lambda(z) \\
\lambda(x) & \wedge \lambda(y) \wedge \lambda(z) \\
(0.2+\lambda(x)) & \wedge(0.6+\lambda(y)) \wedge \lambda(z)
\end{array}\right)
$$

For $0.2<r \leq 0.3$, int $_{\mathcal{U}_{i n t}}(\lambda, r)=\left(\bigwedge_{y \in X}\left(u_{\rho \odot \rho}(y, x) \rightarrow \lambda(x)\right)\right) \vee\left(\bigwedge_{y \in X}\left(u_{\rho}(y, x) \rightarrow\right.\right.$ $\lambda(x)))=$

$$
\left(\begin{array}{rl}
\lambda(x) & \wedge(0.4+\lambda(y)) \wedge \lambda(z) \\
\lambda(x) & \wedge \lambda(y) \wedge \lambda(z) \\
(0.4+\lambda(x)) & \wedge(0.6+\lambda(y)) \wedge \lambda(z)
\end{array}\right)
$$

For $0<r \leq 0.2$, int $\left._{\mathcal{U}_{\text {int }}}(\lambda, r)=\bigwedge_{y \in X}\left(u_{\rho} \odot u_{\rho}\right)(y, x) \rightarrow \lambda(x)\right)=$

$$
\left(\begin{array}{c}
\lambda(x) \wedge(0.8+\lambda(y)) \wedge \lambda(z) \\
\lambda(x) \wedge \lambda(y) \wedge \lambda(z) \\
(0.4+\lambda(x)) \wedge \lambda(z)
\end{array}\right)
$$

Since $\operatorname{int}_{\mathcal{U}_{i n t}}(\rho, r)=\rho$ if $0.3<r \leq 0.6$ and $\operatorname{int}_{\mathcal{U}_{i n t}}(\rho \odot \rho, r)=\rho \odot \rho$ if $0.2<r \leq$ 0.3 , we have int $_{\mathcal{U}_{\text {int }}} \geq i n t$.

\section{References}

[1] R. Badard, A.A. Ramadan, A.S. Mashhour, Smooth preuniform and proximity spaces, Fuzzy Sets and Systems, 59 (1993), 95-107.

[2] R. Bělohlávek, Fuzzy Relational Systems, Kluwer Academic Publishers, New York, (2002), doi: 10.1007/978-1-4615-0633-1.

[3] D. Čimoka, A.P. Šostak, L-fuzzy syntopogenous structures, Part I: Fundamentals and application to L-fuzzy topologies, L-fuzzy proximities and L-fuzzy uniformities, Fuzzy Sets and Systems, 232 (2013), 74-97.

[4] Fang Jinming, I-fuzzy Alexandrov topologies and specialization orders, Fuzzy Sets and Systems, 158(2007), 2359-2374, doi: 10.1016/j.fss.2007.05.001.

[5] P. Hájek, Metamathematices of Fuzzy Logic, Kluwer Academic Publishers, Dordrecht (1998), doi: 10.1007/978-94-011-5300-3.

[6] U. Höhle, S.E. Rodabaugh, Mathematics of Fuzzy Sets: Logic, Topology, and Measure Theory, The Handbooks of Fuzzy Sets Series 3, Kluwer Academic Publishers, Boston, 1999, doi: 10.1007/978-1-4615-5079-2.

[7] B. Hutton, Uniformities in fuzzy topological spaces, J. Math. Anal. Appl., 58 (1977), 74-79. doi: $10.1016 / 0022-247 x(77) 90192-5$.

[8] A.K. Katsaras, On fuzzy uniform spaces, J. Math. Anal. Appl., 101, 1984, 97-113. doi: 10.1016/0022-247x(84)90060-x. 
[9] Y.C. Kim, Y.S. Kim, $L$-approximation spaces and $L$-fuzzy quasi-uniform Spaces, Information Sciences, 179 (2009), 2028-2048.

[10] R. Lowen, Fuzzy uniform spaces, J. Math. Anal. Appl., 82 (1981), 370-385, doi: $10.1016 / 0022-247 x(81) 90202-x$.

[11] H. Lai, D. Zhang, Fuzzy preorder and fuzzy topology, Fuzzy Sets and Systems, 157 (2006), 1865-1885, doi: 10.1016/j.fss.2006.02.013.

[12] A.A.Ramadan, E.H.Elkordy, Yong Chan Kim, Perfect $L$-fuzzy topogenous space, $L$-fuzzy quasi-proximities and $L$-fuzzy quasi-uniform spaces, J. Intelligent and Fuzzy Systems $\mathbf{2 8}$ (2015), 2591-2604, doi: 10.3233/IFS-151538.

[13] A.A.Ramadan, E.H.Elkordy, Yong Chan Kim, Relationships between $L$-fuzzy quasiuniform structures and L-fuzzy topologies, J. Intelligent and Fuzzy Systems 28 (2015), 2319-2327, doi: 10.3233/IFS-141515.

[14] A.A. Ramadan,Y.C. Kim, M.K. El-Gayyar, On fuzzy uniform spaces, The Journal of Fuzzy Mathematics, 11 (2003), 279-299.

[15] S.E. Rodabaugh, E.P. Klement, Topological and Algebraic Structures In Fuzzy Sets, The Handbook of Recent Developments in the Mathematics of Fuzzy Sets, Kluwer Academic Publishers, Boston, Dordrecht, London, 2003, doi: 10.1007/978-94-017-0231-7. 